\title{
Clinical and Angiographic Features and Stroke Types in Adult Moyamoya Disease
}

\author{
D.-K. Jang, K.-S. Lee, H.K. Rha, P.-W. Huh, J.-H. Yang, I.S. Park, J.-G. Ahn, J.H. Sung, and Y.-M. Han
}

\section{ABSTRACT}

BACKGROUND AND PURPOSE: This study was conducted to elucidate the association between clinical and angiographic characteristics and stroke types in adult Moyamoya disease that has been rarely evaluated.

MATERIALS AND METHODS: We analyzed the clinical and radiologic data obtained from a retrospective adult Moyamoya disease cohort with acute strokes, which were classified into 7 categories: large-artery infarct, hemodynamic infarct, perforator infarct, deep intracerebral hemorrhage, lobar intracerebral hemorrhage, intraventricular hemorrhage, and SAH. With conventional angiography, which was performed in the hemispheres with acute strokes, the Suzuki angiographic stage, intracranial aneurysm, major artery occlusion, and collateral vessel development were confirmed within 1 month of stroke onset.

RESULTS: This study included 79 patients with acute ischemic stroke and 96 patients with acute hemorrhagic stroke. The angiographic stage had a strong tendency to be more advanced in the hemorrhagic than the ischemic patients $(P=.061)$. Intracranial aneurysms were more frequently found in the hemorrhagic than ischemic or control hemispheres $(P=.002)$. Occlusions of the anterior cerebral artery and development of fetal-type posterior cerebral artery were more frequently observed in the hemorrhagic than the ischemic $(P=.001$ and .01 , respectively) or control hemispheres $(P=.011$ and .013 , respectively). MCA occlusion $(P=.039)$ and collateral flow development, including the ethmoidal Moyamoya vessels $(P=.036)$ and transdural anastomosis of the external carotid artery $(P=.022)$, occurred more often in the hemorrhagic than the ischemic hemispheres. Anterior cerebral artery occlusion occurred more frequently in patients with deep intracerebral hemorrhage or intraventricular hemorrhage than with lobar intracerebral hemorrhage $(P=.009)$.

CONCLUSIONS: In adult Moyamoya disease, major artery occlusion and collateral compensation occurred more often in the hemorrhagic than in the ischemic hemispheres. Thus, anterior cerebral artery occlusion with or without MCA occlusion and intracranial aneurysms may be the main contributing factors to hemorrhagic stroke in adult patients with Moyamoya disease.

ABBREVIATIONS: $\mathrm{ACA}=$ anterior cerebral artery; $\mathrm{ECA}=$ external carotid artery; $\mathrm{HI}=$ hemodynamic infarct; $\mathrm{IVH}=$ intraventricular hemorrhage; $\mathrm{LAI}=$ large-artery infarct; $\mathrm{MMD}=$ Moyamoya disease; $\mathrm{PCA}=$ posterior cerebral artery; $\mathrm{PI}=$ perforator infarct

M oyamoya disease (MMD) is a chronic, progressive cerebrovascular disorder with bilateral stenosis or occlusion of the supraclinoid ICA and its major branches at the surrounding area of the circle of Willis. ${ }^{1}$ MMD exhibits diverse symptoms, such as

Received August 8, 2013; accepted after revision October 21

From the Department of Neurosurgery (D.-K.J., Y.-M.H.), Incheon St. Mary's Hospital, College of Medicine, The Catholic University of Korea, Incheon, Korea; Department of Neurosurgery (K.-S.L.), Seoul St. Mary's Hospital, College of Medicine, The Catholic University of Korea, Seoul, Korea; Department of Neurosurgery (H.K.R.), Yeouido St. Mary's Hospital, College of Medicine, The Catholic University of Korea, Seoul, Korea; Department of Neurosurgery (P.-W.H.), Uijeongbu St. Mary's Hospital, College of Medicine, The Catholic University of Korea, Uijeongbu, Korea; Department of Neurosurgery (J.-H.Y.), Daejeon St. Mary's Hospital, College of Medicine, The Catholic University of Korea, Daejeon, Korea; Department of Neurosurgery (I.S.P.), Bucheon St. Mary's Hospital, College of Medicine, The Catholic University of Korea, Bucheon, Korea; Department of Neurosurgery (J.-G.A.), St. Paul's Hospital, College of Medicine, The Catholic University of Korea, Seoul, Korea; and Department of Neurosurgery (J.H.S.), St. Vincent's Hospital, College of Medicine, The Catholic University of Korea, Suwon, Korea.
TIA, recurrent TIAs, cerebral infarct, intracranial hemorrhage, headache, seizure, cognitive decline, ataxia, or choreiform movements; it can also be asymptomatic. In adult MMD, approximately half of the patients have intracranial hemorrhage, and the rest of the patients have TIA or cerebral infarct. ${ }^{2,3}$ Recently, MMD was reported to exhibit a bimodal peak of symptom onset in the ischemic type and a unimodal peak in the hemorrhagic type. ${ }^{4}$ However, the cause of differential onset and the distinct presentation in MMD are not completely understood. Although angio-

This study was supported by a grant obtained from the Korea Health 21 R\&D Project (A070001), Ministry of Health and Welfare, Republic of Korea.

Please address correspondence to Kwan-Sung Lee, MD, PhD, Department of Neurosurgery, Seoul St. Mary's Hospital, The Catholic University of Korea, College of Medicine, 222 Banpo-Daero, Seocho-Gu, Seoul, Korea, 137-701; e-mail: nslk@catholic.ac.kr

- Indicates open access to non-subscribers at www.ajnr.org

http://dx.doi.org/10.3174/ajnr.A3819 
graphic factors causing intracranial hemorrhage in adult MMD, such as dilation and branch extension of the anterior choroidal artery or posterior communicating artery have also been reported, ${ }^{5-7}$ direct comparison studies on the angiographic features according to stroke type in adult MMD have been limited.

We hypothesized that there may be differences in major artery occlusion and collateral patterns according to stroke type. Because understanding differences in the angiographic characteristics of ischemic and hemorrhagic stroke types may be helpful to prevent future strokes and make a good prognosis in adult patients with MMD, this study aimed to compare the angiographic features of adult MMD with ischemic and hemorrhagic stroke.

\section{MATERIALS AND METHODS}

From 2002-2011, a retrospective adult MMD cohort in 8 hospitals was recruited. The study protocol was approved by the Institutional Review Board of Catholic Medical Center at the Catholic University of Korea. The inclusion criteria were as follows: 1) age $\geq 18$ years, 2) compatible with the Japanese MMD diagnosis guideline, ${ }^{1} 3$ ) radiologically proven symptomatic acute stroke, 4) digital subtraction angiography that had been performed within 1 month of the acute stroke, and 5) newly diagnosed MMD. Patients with Moyamoya syndrome or probable MMD, ethnicity other than Korean, incomplete radiologic data, or a term between acute stroke and DSA longer than 1 month were excluded. Those who had had repeat strokes at diagnosis or new strokes after surgical treatment were also excluded. The baseline characteristics were scrutinized, such as age at the initial diagnosis of MMD; sex; the presence of hypertension or antihypertensive medication history; the presence of diabetes mellitus or antidiabetic medication history; clinical symptoms, including headaches, weakness, sensory changes, visual disturbances, seizures, and decreased consciousness; initial Glasgow coma scale score; and mRS.

An ischemic stroke was confirmed by use of MR DWI, and a hemorrhagic stroke was identified by means of CT, T2WI, or gradient-echo MR imaging of abnormal lesions, with a low signal indicating a hemosiderin rim that was larger than $0.5 \mathrm{~cm}$. Acute ischemic strokes were categorized into 6 groups, as suggested by Mugikura et $\mathrm{al}^{8}$ and Kim et $\mathrm{al}^{9}$ : anterior cerebral artery (ACA) infarct; anterior watershed infarct between the ACA and MCA territories; anterior half of the MCA territory infarct; posterior half of MCA territory infarct; posterior watershed infarct between the MCA and posterior cerebral artery (PCA) territories; PCA infarct; and perforator infarct (PI). Acute cerebral hemorrhages were classified as follows: frontal lobe hemorrhage, temporal lobe hemorrhage, parietal lobe hemorrhage, occipital lobe hemorrhage, basal ganglia hemorrhage, thalamic hemorrhage, intraventricular hemorrhage (IVH), and SAH.

Angiographic details of all the hemispheres included the following: angiographic stage as suggested by Suzuki and Takaku ${ }^{10}$; ACA, MCA, and PCA occlusions; fetal PCA development (enlargement of the posterior communicating artery); ethmoidal Moyamoya development; leptomeningeal collateral from the PCA to the ACA or MCA territories; collateral flow through the posterior communicating artery from the PCA to the ICA; transdural anastomosis from the external carotid artery (ECA); and cerebral aneurysm (Fig 1). Two independent neurologic physicians reviewed all the radiologic data. When a disagreement regarding the diagnosis and measurement between reviewers occurred, a consensus was drawn after a thorough discussion.

There were 372 adult patients with MMD from 2002-2011. Sixty-four patients $(17.2 \%)$ were excluded because of incomplete radiologic data $(n=60)$ and other ethnicity $(n=4 ; 3$ Chinese and 1 Japanese). Among the remaining 308 study entrants, 130 $(42.2 \%)$ were excluded because of presenting symptoms of chronic or recurrent stroke $(n=71,23.1 \%)$, TIA $(n=40,12.9 \%)$, headache $(n=9,2.9 \%)$, dizziness $(n=4,1.3 \%)$, seizure $(n=1$, $0.3 \%)$, and an absence of symptoms $(n=5,1.6 \%)$. Finally, 178 patients $(57.8 \%)$ who had acute stroke and underwent DSA within 1 month from ictus were included. One patient with ruptured basilar apex aneurysm, 1 patient with pure IVH, and 1 patient with nonaneurysmal SAH were excluded from the final analysis because of an inability to define the laterality of the bleeding site.

\section{Statistics}

To compare the angiographic characteristics between acute ischemic stroke and hemorrhagic stroke in adult MMD, the stroke subtypes were grouped as follows: ACA infarct, anterior or posterior half of an MCA infarct and a PCA infarct into a large-artery infarct; PI; anterior watershed and posterior watershed infarct into a hemodynamic infarct (HI); basal ganglia or thalamic hemorrhage into a deep intracerebral hemorrhage; frontal, temporal, or parietal lobe hemorrhage into a lobar intracerebral hemorrhage; IVH; and SAH. To confirm the laterality of strokes, in cases of pure IVH, the stroke hemisphere was defined as the side of the lateral ventricle with the larger hemorrhage volume. The baseline characteristics and angiographic parameters between the ischemic and hemorrhagic hemispheres were compared by means of the $\chi^{2}$ or Fisher exact tests for categoric variables and the $t$ test for continuous variables. The Mann-Whitney $U$ test was performed for non-normally distributed continuous variables. Twotailed tests were used in all statistical analyses. Statistical significance was established at a value of $P \leq .05$. All statistical analyses were performed by use of SAS version 9.2 (SAS Institute, Cary, North Carolina).

\section{RESULTS}

\section{Patient Characteristics}

The demographic data of 175 adult patients with MMD with acute stroke at diagnosis are summarized in Table 1. The median age at diagnosis was 43 years (range, 18-78 years). The male-to-female ratio was 1 to 1.57 . Of the 175 patients, $44(25.1 \%)$ had a history of hypertension, and $20(11.4 \%)$ had diabetes. The most common symptoms of stroke were weakness $(40.6 \%)$, decreased consciousness (35.4\%), headache (29.7\%), speech disturbance (23.4\%), visual disturbance $(9.1 \%)$, sensory change (5.1\%), and seizure (2.9\%). An initial Glasgow Coma Scale score $>12$ was observed in 133 patients (76\%), and an initial $\mathrm{mRS}<3$ was observed in 100 patients $(57.1 \%)$. The angiographic stages were as follows: stage 1 in 29 hemispheres $(8.3 \%)$, stage 2 in 87 hemispheres (24.9\%), stage 3 in 137 hemispheres $(39.1 \%)$, stage 4 in 65 hemispheres (18.6\%), stage 5 in 29 hemispheres $(8.3 \%)$, and stage 6 in 3 hemispheres $(0.9 \%)$. Nine intracranial aneurysms were identified (5 ruptured and 4 unruptured). Seventy-nine patients $(45.1 \%)$ had ischemic stroke and 96 patients 



FIG 1. Representative radiologic and angiographic features of adult Moyamoya disease with ischemic $(A-E)$ and hemorrhagic $(F-J)$ stroke. Ischemic MMD shows right frontal large-artery infarct $(A)$ with MCA occlusion (B) but without anterior cerebral artery occlusion (C) and good collateral flows from both posterior cerebral arteries (D) but without transdural anastomosis from the right external carotid artery (E). Hemorrhagic MMD shows left intraventricular hemorrhage (F), with ACA, MCA and PCA occlusion $(G-l)$, with lenticulostriate artery aneurysm ( $G$ and $H$, white arrows) and the development of ethmoidal Moyamoya vessels $(H)$, fetal-type PCA $(H)$, and transdural anastomosis from the ECA (, black arrow).

(54.9\%) had hemorrhagic stroke. Between the ischemic and hemorrhagic stroke groups, there was no significant difference regarding hypertension; however, diabetes occurred more frequently in the ischemic stroke group $(17.7 \%)$ than in the hemorrhagic stroke group (6.3\%) $(P=.018)$. With respect to symptom presentation, headache and consciousness deterioration occurred more frequently in the hemorrhagic stroke group than in the ischemic stroke group $(P=$ .002 and $P<.001$, respectively). However, sensory changes as well as speech and visual disturbances were more frequently observed in the ischemic stroke group than in the hemorrhagic stroke group $(P=$ $.012, P<.001$, and $P<.001$, respectively). The prevalence rates for weakness and seizure were not different between the 2 groups. The 
Table 1: Clinical summary of 175 adult patients with Moyamoya disease with acute ischemic or hemorrhagic stroke

\begin{tabular}{|c|c|c|c|c|}
\hline Variables & $\begin{array}{c}\text { Total } \\
(n=175)\end{array}$ & $\begin{array}{c}\text { Infarct group } \\
(n=79)\end{array}$ & $\begin{array}{c}\text { Hemorrhage Group } \\
(n=96)\end{array}$ & $P$ Value \\
\hline \multicolumn{5}{|l|}{ Demographics } \\
\hline Age, y (mean $\pm S D)$ & $42.2 \pm 11.3$ & $41.2 \pm 11.2$ & $43.1 \pm 11.3$ & .28 \\
\hline Female & 107 (61.1) & $46(58.2)$ & $61(63.5)$ & .47 \\
\hline Family history & $1(0.6)$ & $1(1.3)$ & 0 & .45 \\
\hline \multicolumn{5}{|l|}{ Vascular risk factors } \\
\hline Hypertension & $44(25.1)$ & $22(27.8)$ & $22(22.9)$ & .45 \\
\hline Diabetes & $20(11.4)$ & $14(17.7)$ & $6(6.3)$ & .018 \\
\hline \multicolumn{5}{|l|}{ Symptoms } \\
\hline Headache & $52(29.7)$ & $14(17.7)$ & $38(39.6)$ & .002 \\
\hline Weakness & $71(40.6)$ & $33(41.8)$ & $38(39.6)$ & .76 \\
\hline Sensory change & $9(5.1)$ & $8(10.1)$ & $1(1.0)$ & .012 \\
\hline Speech disturbance & $41(23.4)$ & $30(38.0)$ & $11(11.5)$ & $<.001$ \\
\hline Visual disturbance & $16(9.1)$ & $15(19.0)$ & $1(1.0)$ & $<.001$ \\
\hline Seizure & $5(2.9)$ & $1(1.3)$ & $4(4.2)$ & .38 \\
\hline Decreased consciousness & $62(35.4)$ & $4(5.1)$ & $58(60.4)$ & $<.001$ \\
\hline \multicolumn{5}{|l|}{ Initial neurology } \\
\hline Initial GCS $(>12)$ & $133(76.0)$ & 78 (98.7) & $55(57.3)$ & $<.001$ \\
\hline Initial mRS $(<3)$ & $100(57.1)$ & $55(69.6)$ & $45(46.9)$ & .002 \\
\hline Suzuki stage ( 350 hemispheres) & & & & .061 \\
\hline 1 & $29(8.3)$ & $19(12.0)$ & $10(5.2)$ & \\
\hline 2 & $87(24.9)$ & $46(29.1)$ & $41(21.4)$ & \\
\hline 3 & $137(39.1)$ & $53(33.5)$ & $84(43.8)$ & \\
\hline 4 & $65(18.6)$ & $26(16.5)$ & $39(20.3)$ & \\
\hline 5 & $29(8.3)$ & $13(8.2)$ & $16(8.3)^{\prime}$ & \\
\hline 6 & $3(0.9)$ & $1(0.6)$ & $2(1.0)$ & \\
\hline
\end{tabular}

Note:-SD indicates standard deviation; GCS, Glasgow Coma Scale.

Table 2: Distribution of intracranial aneurysms ${ }^{a}$ according to acute stroke subtype in $\mathbf{3 5 0}$ Moyamoya hemispheres

\begin{tabular}{|c|c|c|c|c|c|}
\hline & $\begin{array}{l}\text { Ischemic Stroke } \\
(n=85)\end{array}$ & $\begin{array}{l}\text { Hemorrhagic } \\
\text { Stroke }(n=96)\end{array}$ & $\begin{array}{l}\text { Silent Stroke } \\
\quad(n=36)\end{array}$ & $\begin{array}{c}\text { None } \\
(n=133)\end{array}$ & $P$ Value \\
\hline Bleeding & & & & & .002 \\
\hline $\begin{array}{l}\text { Ruptured } \\
\text { Suzuki stage }\end{array}$ & 0 & 5 & 0 & 0 & \\
\hline 2 & & 2 & & & \\
\hline 3 & & 3 & & & \\
\hline $\begin{array}{l}\text { Unruptured } \\
\text { Suzuki stage }\end{array}$ & 1 & 3 & 0 & 0 & \\
\hline 3 & & 1 & & & \\
\hline 5 & 1 & 2 & & & \\
\hline Location & & & & & \\
\hline Anterior circulation & & 5 & 0 & 0 & \\
\hline Posterior circulation & & 4 & 0 & 0 & \\
\hline
\end{tabular}

${ }^{a}$ One aneurysm was identified in the infarct hemisphere (unruptured anterior falx artery aneurysm), and 8 aneurysms were identified in the hemorrhage hemispheres, consisting of 5 ruptured aneurysms (1 at the splenial artery, 2 at the anterior choroidal artery, 1 at the lenticulostriate artery, and 1 at the posterior cerebral artery) and 3 unruptured aneurysms ( 2 at the basilar apex and 1 at the superior cerebellar artery).

initial neurologic status (Glasgow Coma Scale and mRS) was better in the ischemic stroke group than in the hemorrhagic stroke group $(P<.001$ and .002 , respectively $)$.

\section{Stroke and Angiographic Profiles}

Acute strokes were identified in 181 hemispheres with 85 ischemic strokes ( 6 bilateral) and 96 hemorrhagic strokes among 350 hemispheres in 175 patients. In addition, 36 hemispheres with silent strokes with abnormal signal changes on T1 or T2 MR imaging were observed, and the remaining 133 hemispheres (control hemispheres) did not show any symptoms or abnormal lesions on brain MR or CT. Representative adult patients with MMD with acute ischemic or hemorrhagic stroke are illustrated in Fig 1. There were significantly more intracranial aneurysms in the hemorrhagic hemisphere than the control or ischemic hemisphere $(P=.002)$ (Table 2). The proportions of acute stroke were as follows: LAI in 37 hemispheres (20.4\%), HI in 35 hemispheres (19.3\%), PI in 13 hemispheres $(7.2 \%)$, deep intracerebral hemorrhage in 50 hemispheres (27.6\%), IVH in 32 hemispheres (17.7\%), and lobar intracerebral hemorrhage in 14 hemispheres (7.7\%) (Fig 2A). The ratio of LAI to HI or PI was 1:1.3, and the ratio of deep intracerebral hemorrhage or IVH to lobar intracerebral hemorrhage was 5.9:1 (Tables 3 and 4). The angiographic stage was significantly more advanced in the hemorrhagic than the control hemispheres and markedly tended to be more progressed in the hemorrhagic than the ischemic hemispheres (Fig 2C). In the hemorrhagic hemispheres, ACA occlusion was more frequently observed compared with the ischemic and control hemispheres $(P=$ .001 and .01 , respectively) (Fig $3 A,-C$ ). MCA occlusion was also more frequent in the hemorrhagic than the ischemic hemisphere (.039). Hemorrhagic hemispheres were more advanced than ischemic or control hemispheres with regard to ACA and MCA occlusion patterns $(P=.034$ and .001 , respectively) (Fig $3 C$ ). In addition, collateral flows, including ethmoidal Moyamoya and transdural anastomosis from the ECA, were more common in the hemorrhagic hemisphere than in the ischemic hemisphere $(P=.036$ and .022 , respectively) (Fig $3 E,-I$ ). Fetal PCA was also more frequently observed in the hemorrhagic hemisphere than in the control and ischemic hemispheres $(P=.001$ and .011 , respectively) (Fig $3 F$ ).

For the subgroup analysis, according to the topographic patterns of acute stroke, acute ischemic strokes were dichotomized into LAI (37) and HI or PI (48), and acute hemorrhagic strokes were classified into deep intracerebral hemorrhage or IVH (82) and lobar intracerebral hemorrhage (14). ACA, MCA, and PCA occlusions were found more frequently in the LAI group than in the HI or PI group $(P=.009$, .031 , and .003 , respectively), but leptomeningeal collateral flow from the PCA to the ACA or MCA occurred more frequently in the HI or PI group than in the LAI group $(P=.001)$ (Table 3$)$. ACA occlusion, PCA occlusion, ethmoidal Moyamoya, and transdural anastomosis from the ECA occurred more often in posterior circulation than in anterior circulation ischemic strokes $(P=$ $.027, P<.001, P=.026$, and $P<.001$, respectively) (Table 3 ). In the hemorrhagic hemispheres, only an ACA occlusion was observed more frequently in the deep intracerebral hemorrhage or 


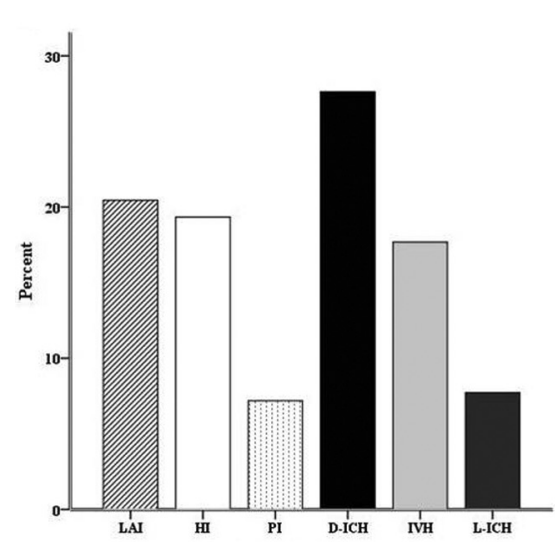

A

Acute Stroke Type

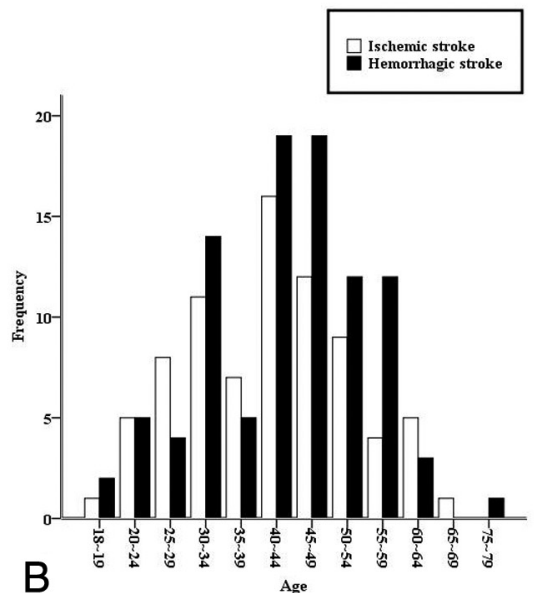

B

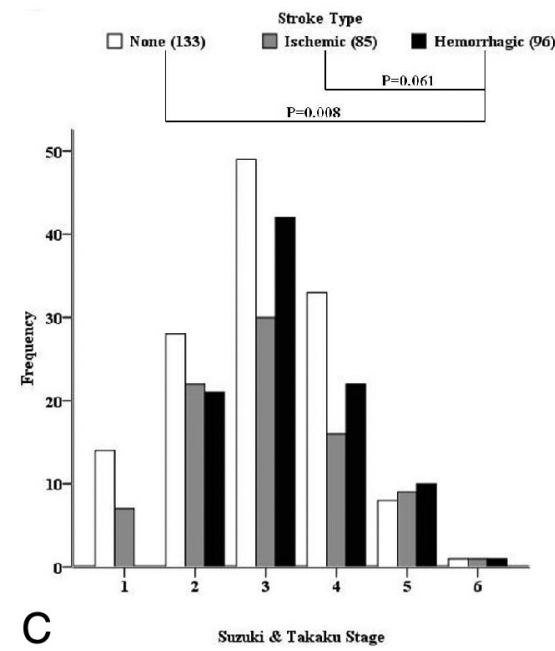

FIG 2. Topographic patterns of acute stroke $(A)$, age distribution of adult Moyamoya disease according to stroke type $(B, P=.28)$, and angiographic distribution of stroke type (C).

Table 3: Angiographic differences according to topographic patterns of acute infarct in adult Moyamoya disease

\begin{tabular}{|c|c|c|c|c|c|c|}
\hline \multirow[b]{2}{*}{ Variables } & \multicolumn{2}{|c|}{ LAI Versus HI or PI } & \multirow[b]{2}{*}{$P$ Value } & \multicolumn{2}{|c|}{ Anterior Versus Posterior } & \multirow[b]{2}{*}{$P$ Value } \\
\hline & LAI $(n=37)$ & HI or PI $(n=48)$ & & $\begin{array}{l}\text { Anterior Circulation } \\
\qquad(n=57)\end{array}$ & $\begin{array}{l}\text { Posterior Circulation } \\
\qquad(n=28)\end{array}$ & \\
\hline ACA occlusion & $30(81.1)$ & $26(54.2)$ & .009 & $33(57.9)$ & $23(82.1)$ & .027 \\
\hline MCA occlusion & $31(83.8)$ & $30(62.5)$ & .031 & $39(68.4)$ & $22(78.6)$ & .32 \\
\hline PCA occlusion & $18(48.6)$ & $9(18.8)$ & .003 & $11(19.3)$ & $16(57.1)$ & $<.001$ \\
\hline Ethmoidal Moyamoya & $12(32.4)$ & $14(29.2)$ & .74 & $13(22.8)$ & $13(46.4)$ & .026 \\
\hline Fetal-type PCA & $12(32.4)$ & $18(37.5)$ & .63 & $21(36.8)$ & $9(32.1)$ & .67 \\
\hline PCA collateral & $23(62.2)$ & 44 (91.7) & .001 & $47(82.5)$ & $20(71.4)$ & .24 \\
\hline PcomA collateral & $3(8.1)$ & $2(4.2)$ & .65 & $3(5.3)$ & $2(7.1)$ & .72 \\
\hline ECA collateral & $16(43.2)$ & $13(27.1)$ & .12 & $12(21.1)$ & $17(60.7)$ & $<.001$ \\
\hline
\end{tabular}

Note:-Ethmoidal Moyamoya, fetal-type PCA, PCA collateral, PcomA collateral, and ECA collateral indicate the development of ethmoidal Moyamoya vessels, enlargement of PcomA or showing blood supply to PCA territories through PcomA, leptomeningeal collateral vessels from PCA to ACA or MCA territories, collateral flow from PCA to ICA through PcomA, and transdural anastomosis from ECA to ICA, respectively.

PcomA indicates posterior communicating artery.

Table 4: Angiographic difference according to the location of the acute hemorrhage in adult MMD

\begin{tabular}{lccc}
\hline \multicolumn{1}{c}{ Variables } & $\begin{array}{c}\text { D-ICH or IVH } \\
(\boldsymbol{n}=\mathbf{8 2})\end{array}$ & $\begin{array}{c}\text { L-ICH } \\
(\boldsymbol{n}=\mathbf{1 4})\end{array}$ & $\boldsymbol{P}$ Value \\
\hline ACA occlusion & $74(90.2)$ & $9(64.3)$ & .009 \\
MCA occlusion & $70(85.4)$ & $11(78.6)$ & .45 \\
PCA occlusion & $18(22.0)$ & $3(21.4)$ & .99 \\
Ethmoidal Moyamoya & $39(47.6)$ & $5(35.7)$ & .41 \\
Fetal-type PCA & $46(56.1)$ & $6(42.9)$ & .36 \\
PCA collateral & $69(84.1)$ & $11(78.6)$ & .69 \\
PcomA collateral & $6(7.3)$ & $1(7.1)$ & .99 \\
ECA collateral & $44(53.7)$ & $5(35.7)$ & .21 \\
Aneurysm $^{\text {a }}$ & $5(6.1)$ & $2(14.3)$ & .27 \\
\hline
\end{tabular}

Note:-Ethmoidal Moyamoya, fetal-type PCA, PCA collateral, PcomA collateral, and ECA collateral indicate the development of ethmoidal Moyamoya vessels, enlargement of PcomA or showing blood supply to PCA territories through PcomA, leptomeningeal collateral vessels from PCA to ACA or MCA territories, collateral flow from PCA to ICA through PcomA, and transdural anastomosis from ECA to ICA, respectively.

$\mathrm{D}-\mathrm{ICH}$ indicates deep intracerebral hemorrhage; $\mathrm{L}-\mathrm{ICH}$, lobar intracerebral hemorrhage; PcomA, posterior communicating artery.

a Two aneurysms were identified simultaneously in 1 patient in the D-ICH or IVH group.

IVH group than in the lobar intracerebral hemorrhage group $(P=.009)$ (Table 4$)$.

\section{DISCUSSION}

This study demonstrated that the hemorrhagic hemisphere in adult MMD was associated more with intracranial aneurysms,
ACA occlusion, and fetal-type PCA development than the ischemic or control hemispheres. MCA occlusion and collateral flow, such as ethmoidal Moyamoya vessels and transdural anastomosis from the ECA, were detected more often in the hemorrhagic than ischemic hemispheres. ACA occlusion was associated with deep intracerebral hemorrhage or IVH.

As independent risk factors for hemorrhagic stroke in MMD, anterior choroidal artery and posterior communicating artery dilation or extension have been described. ${ }^{5-7}$ Moreover, cerebral microbleeds in adult patients with MMD were reported to be probable predictors of subsequent hemorrhage, and dilation and extension of the anterior choroidal artery or posterior communicating artery were independent risk factors of cerebral microbleeds in deep and periventricular white matter. ${ }^{11}$ In our study, fetal-type PCA development was more commonly observed in the hemorrhagic hemispheres than in the ischemic or control hemispheres. In addition, we newly described the association of ACA occlusion and hemorrhagic stroke (Fig $3 A,-C$ ). Intracranial aneurysms were also proven to be an independent risk factor for hemorrhagic stroke in adult patients with MMD (Table 2). The more common prevalence of transdural anastomosis from ECA and ethmoidal Moyamoya vessel development in hemorrhagic hemispheres represent newly detected findings in our study (Fig $3 E,-I)$. In the subgroup analysis, ACA occlusion and deep intra- 

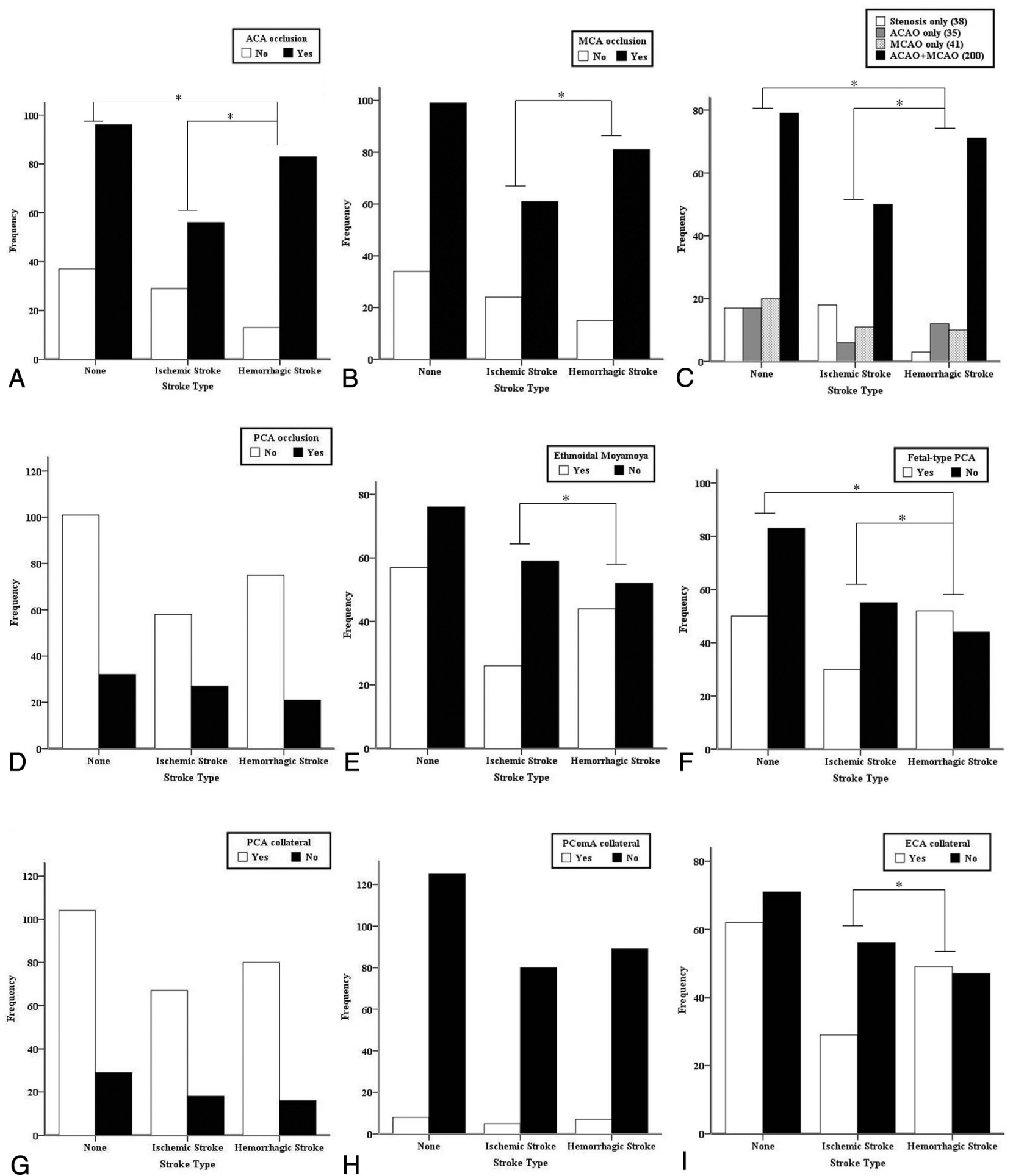

FIG 3. Angiographic patterns according to acute stroke type of 314 hemispheres in 175 adult patients with Moyamoya disease. Asterisks indicate statistical significances between the respective hemispheres ( $A-C, E, F$, and I).

cerebral hemorrhage or IVH occurrence were associated with each other (Table 4). Taken together, these findings may show important differences between ischemic and hemorrhagic adult MMD.

However, some researchers have reported that ischemic childhood MMD and most ischemic strokes might be associated with the degree of steno-occlusive changes in the ICA and PCA. ${ }^{8,12}$ In ischemic adult MMD, major artery occlusion, including ACA, MCA, and PCA occlusions, was associated more with LAI and posterior circulation ischemic stroke than with $\mathrm{HI}$ or PI and with anterior circulation ischemic stroke (Table 3). Therefore, adult ischemic MMD may have angiographic characteristics similar to those in pediatric MMD with ischemic stroke.

The hemorrhagic hemispheres in adult MMD showed more 
advanced angiographic stages (Fig 2C) than did the control hemispheres and demonstrated more progressive features (Fig 3 ) than did the ischemic hemispheres, particularly for ACA occlusion, MCA occlusion, ethmoidal Moyamoya, fetal-type PCA, and transdural anastomosis from the ECA. These characteristics may be important angiographic factors in adult MMD and are consistent with the findings of previous studies. ${ }^{5,13}$ Specifically, Morioka et $\mathrm{al}^{5}$ demonstrated that in $\mathrm{MMD}$, ischemic and hemorrhagic hemispheres in both pediatric and adult patients differed at the site of supraclinoid ICA occlusion or stenosis from the ophthalmic segment to the ICA bifurcation. The present study suggests that hemodynamic stress may differ between the ischemic and hemorrhagic hemispheres in adult MMD. Independent of MCA occlusion, ACA occlusion was more frequently detected in the hemorrhagic hemisphere than in the ischemic hemisphere (Fig 3C), and intracranial aneurysms were more frequently detected in hemorrhagic MMD. According to the stroke subtype, the hemorrhagic hemispheres also had more bleeding sites near the midline structure or deep brain area than in the subcortical and cortical areas (Table 4).

Such angiographic differences in ischemic and hemorrhagic MMD might originate from genetic differences. With the identification of the first MMD gene, RNF213, rapid progress has been made in genetic research on MMD. ${ }^{14}$ Miyatake et al ${ }^{13}$ revealed that in the homozygote c.14576G>A variant of RNF213, 60\% of patients were diagnosed with MMD before the age of 4 years, and all had ischemic strokes. They also showed that patients with homozygotes had a significantly earlier age at onset than those of the heterozygote or wild-type c.14576G $>$ A variants. According to their results, there may be large proportion of heterozygote (GA) or wild-type (GG) c.14576G >A variants in RNF213 in patients with adulthood-onset MMD. ${ }^{13}$ Unfortunately, there is still no report on the c.14576G $>$ A variant of RNF213 with MMD in Korea. Further genetic correlation studies with the angiographic and clinical findings of patients with Moyamoya in Korea may help us to understand the causes of different stroke types. Regarding the stroke risk factors in our cohort, there was a high incidence of diabetes but not of hypertension in ischemic MMD compared with hemorrhagic MMD (Table 1). A future study on whether general risk factors, such as diabetes, affect stroke occurrence in adult patients with MMD is necessary. Although our results did not reveal a difference in the age distribution between ischemic and hemorrhagic strokes in adult MMD (Table 1 and Fig 2B), there have been few studies reporting adult MMD in which the symptoms cross over. In addition, most of the symptoms occurred in the ischemic stroke after a hemorrhagic stroke. ${ }^{9,15,16}$ These findings suggest that different stroke presentations in adult MMD may be associated with inherent factors.

Bypass surgery may be helpful in reducing the risk of stroke development in adulthood-onset ischemic MMD, similar to childhood-onset MMD, whereas the effectiveness of bypass surgery for stroke prevention is still controversial in adult MMD with intracranial hemorrhage. ${ }^{17-19}$ An additional study should be performed on whether a difference in the surgical efficacy between the ischemic and hemorrhagic hemispheres in adult patients with MMD may be associated with any respective angiographic differences.
Our study is limited by the representativeness of ischemic MMD, which included only ischemic stroke cases and no TIA cases. Thus, sampling bias may influence the results of this study. Furthermore, the dilation of the anterior choroidal artery was not assessed as a predictor of hemorrhagic stroke in adult MMD. An additional weakness of this study was that it only demonstrated an association and not causality between the angiographic differences and stroke types.

\section{CONCLUSIONS}

Most hemorrhagic strokes in adult MMD occurred near the midline or deep brain structures, in contrast to ischemic strokes. The angiographic status of an acute stroke hemisphere was more advanced in the hemorrhagic than in the ischemic group in adult patients with MMD. Major artery occlusion and collateral compensation occurred more often in the hemorrhagic than in the ischemic hemispheres. Thus, ACA occlusion with or without MCA occlusion and intracranial aneurysms may be main contributing factors to hemorrhagic stroke in adult patients with MMD. We suggest that there may be a potential phenotypic difference between patients with ischemic and hemorrhagic stroke in adult MMD.

\section{ACKNOWLEDGMENTS}

The authors give special thanks to Ye-Kyung Shin and Min-Jung Ham for data coding. Statistical consultation was performed by the Catholic Research Coordinating Center.

Disclosures: Kwan-Sung Lee-UNRELATED: Payment for Manuscript Preparation: For proofreading in English, Comments: \$490 US.

\section{APPENDIX}

Participating hospitals included Bucheon St Mary's Hospital, Daejeon St Mary's Hospital, Incheon St Mary's Hospital, St Paul's Hospital, St Vincent's Hospital, Seoul St Mary's Hospital, Uijeongbu St Mary's Hospital, and Youido St Mary's Hospital (all hospitals were affiliated with the Catholic University of Korea, College of Medicine).

\section{REFERENCES}

1. Fukui M. Guidelines for the diagnosis and treatment of spontaneous occlusion of the circle of Willis ('Moyamoya' disease): Research Committee on Spontaneous Occlusion of the Circle of Willis (Moyamoya Disease) of the Ministry of Health and Welfare, Japan. Clin Neurol Neurosurg 1997;99(Suppl 2):S238-40

2. Han DH, Kwon OK, Byun BJ, et al. A co-operative study: clinical characteristics of 334 Korean patients with Moyamoya disease treated at neurosurgical institutes (1976-1994): The Korean Society for Cerebrovascular Disease. Acta Neurochir (Wien) 2000;142:1263-73

3. Fukui M, Kono S, Sueishi K, et al. Moyamoya disease. Neuropathology 2000;20(Suppl):S61-64

4. Research Committee on the Pathology and Treatment of Spontaneous Occlusion of the Circle of Willis; Health Labour Sciences Research Grant for Research on Measures for Intractable Diseases. Guidelines for diagnosis and treatment of Moyamoya disease (spontaneous occlusion of the circle of Willis). Neurol Med Chir (Tokyo) 2012;52:245-66

5. Morioka M, Hamada J, Kawano T, et al. Angiographic dilatation and branch extension of the anterior choroidal and posterior commu- 
nicating arteries are predictors of hemorrhage in adult Moyamoya patients. Stroke 2003;34:90-95

6. Liu W, Zhu S, Wang X, et al. Evaluation of angiographic changes of the anterior choroidal and posterior communicating arteries for predicting cerebrovascular lesions in adult Moyamoya disease. J Clin Neurosci 2011;18:374-78

7. Irikura K, Miyasaka $\mathrm{Y}$, Kurata A, et al. A source of haemorrhage in adult patients with Moyamoya disease: the significance of tributaries from the choroidal artery. Acta Neurochir (Wien) 1996;138: $1282-86$

8. Mugikura S, Takahashi S, Higano S, et al. The relationship between cerebral infarction and angiographic characteristics in childhood Moyamoya disease. AJNR Am J Neuroradiol 1999;20:336-43

9. Kim DS, Jang DK, Huh PW, et al. Ischaemic stroke after acute intracranial haemorrhage in patients with Moyamoya disease: six new cases and a short literature review. Acta Neurochir (Wien) 2011;153:1253-61

10. Suzuki J, Takaku A. Cerebrovascular "Moyamoya" disease: disease showing abnormal net-like vessels in base of brain. Arch Neurol 1969;20:288-99

11. Sun W, Yuan C, Liu W, et al. Asymptomatic cerebral microbleeds in adult patients with Moyamoya disease: a prospective cohort study with 2 years of follow-up. Cerebrovasc Dis 2013;35:469-75

12. Mugikura S, Higano S, Shirane R, et al. Posterior circulation and high prevalence of ischemic stroke among young pediatric patients with Moyamoya disease: evidence of angiography-based differences by age at diagnosis. AJNR Am J Neuroradiol 2011;32:192-98

13. Miyatake S, Miyake N, Touho H, et al. Homozygous c. 14576G > A variant of RNF213 predicts early-onset and severe form of Moyamoya disease. Neurology 2012;78:803-10

14. Kamada F, Aoki Y, Narisawa A, et al. A genome-wide association study identifies RNF213 as the first Moyamoya disease gene. J Hum Genet 2011;56:34-40

15. Hishikawa T, Tokunaga K, Sugiu K, et al. Clinical and radiographic features of Moyamoya disease in patients with both cerebral ischaemia and haemorrhage. Br J Neurosurg 2013;27:198-201

16. Nagasaka T, Hayashi S, Naito T, et al. Concomitant cerebral infarction and intraventricular hemorrhage in Moyamoya disease: case report. J Neurosurg 2007;106:388-90

17. Starke RM, Komotar RJ, Hickman ZL, et al. Clinical features, surgical treatment, and long-term outcome in adult patients with Moyamoya disease. J Neurosurg 2009;111:936-42

18. Roach ES, Golomb MR, Adams R, et al. Management of stroke in infants and children: a scientific statement from a Special Writing Group of the American Heart Association Stroke Council and the Council on Cardiovascular Disease in the Young. Stroke 2008;39:2644-91

19. Aoki N. Cerebrovascular bypass surgery for the treatment of Moyamoya disease: unsatisfactory outcome in the patients presenting with intracranial hemorrhage. Surg Neurol 1993;40: $372-77$ 